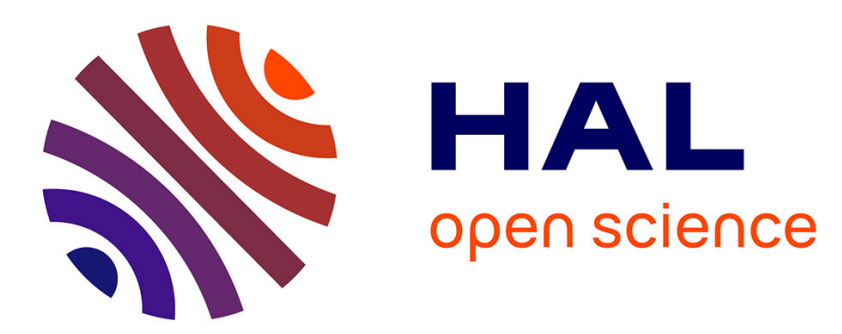

\title{
Stabilization of nonlinear systems in presence of filtered output via extended high-gain observers
}

\author{
Daniele Astolfi, Giacomo Casadei
}

\section{To cite this version:}

Daniele Astolfi, Giacomo Casadei. Stabilization of nonlinear systems in presence of filtered output via extended high-gain observers. Automatica, 2019, 110, pp.108594. 10.1016/j.automatica.2019.108594 . hal-02319700

\author{
HAL Id: hal-02319700 \\ https://hal.science/hal-02319700
}

Submitted on 18 Oct 2019

HAL is a multi-disciplinary open access archive for the deposit and dissemination of scientific research documents, whether they are published or not. The documents may come from teaching and research institutions in France or abroad, or from public or private research centers.
L'archive ouverte pluridisciplinaire HAL, est destinée au dépôt et à la diffusion de documents scientifiques de niveau recherche, publiés ou non, émanant des établissements d'enseignement et de recherche français ou étrangers, des laboratoires publics ou privés. 


\title{
Stabilization of nonlinear systems in presence of filtered output via extended high-gain observers
}

\author{
Daniele Astolfi ${ }^{a}$ and Giacomo Casadei ${ }^{b}$ \\ ${ }^{a}$ Univ Lyon, Université Claude Bernard Lyon 1, CNRS, LAGEPP UMR 5007, 43 boulevard du 11 novembre 1918, F-69100, \\ Villeurbanne, France. \\ ${ }^{\mathrm{b}}$ Laboratoire Ampere Dpt. EEA of the École Centrale de Lyon, Université de Lyon, 69134 Ecully, France.
}

\begin{abstract}
We consider the problem of stabilizing a nonlinear system with filtered output. Given an output feedback control law which satisfies a stability requirement, we consider the case in which the necessary output cannot be measured. The measure is rather the output of an auxiliary stable dynamics in cascade with the system. In place of fully redesign the control architecture, we slightly modify the original control law design by adding a disturbance observer and we recover the desired stability property for the system. The disturbance observer is design as an extended high-gain observer.
\end{abstract}

\section{Introduction}

We consider the problem of output feedback stabilization of single-input single-output nonlinear systems in presence of filtered outputs. In particular, we suppose that the origin of a given system

$$
\Sigma: \begin{cases}\dot{x}=f(x, u), & x \in \mathbb{R}^{n}, u \in \mathbb{R}, \\ y=h(x), & y \in \mathbb{R},\end{cases}
$$

can be stabilized by means of a known (dynamic) output feedback law $\Sigma_{\eta}$

$$
\Sigma_{\eta}:\left\{\begin{array}{l}
\dot{\eta}=\phi(\eta, y), \quad \eta \in \mathbb{R}^{m} \\
u=\psi(\eta, y) .
\end{array}\right.
$$

The design of $\varphi, \psi$ can be obtained with any desired method such as state-feedback design combined with a state observer, feedback linearization approach, backstepping design, forwarding design, Lyapunov redesign, passivity tools, see, e.g., $[13,15,18]$ and references therein. In many practical applications, however, the output $y$ of $\Sigma$ is not directly available, but we can only have access to a static/dynamical version of it. For instance, in $[14,21]$, it is studied the case in which the output $y$ is saturated. In [7], the authors address the

Email addresses: daniele.astolfi@univ-lyon1.fr (Daniele Astolfi), giacomo.casadei@ec-lyon.fr (Giacomo Casadei). case in which the output is multiplied by a time-varying function perturbing its current magnitude. Many other researchers addressed the problem in which the output is subject to sampling, delays, quantization and other hybrid phenomena, see, among others, $[11,26]$.

In this work, we focus to the case in which we can only measure a filtered version of $y$. In particular, $y$ is the input to an auxiliary cascade system $\Sigma_{f}$

$$
\Sigma_{f}: \begin{cases}\dot{z}=A_{r} z+B_{r}(q(z)+y), & z \in \mathbb{R}^{r}, \\ p=C_{r} z, & p \in \mathbb{R}\end{cases}
$$

whose output $p$ is the real available measure. System (3) is supposed to be a stable (in some sense defined in the following) dynamical filter. This scenario can occur when the dynamics of a sensor is slow [12] or drifting [9], as in the context of chemical concentrations sensors, or in parallel power plants applications where the dynamics of a load is modeled as dynamical cascaded systems [5]. These dynamics may represent also a physical interconnection between parts of a system, like transmission line in a network [10]. Our objective is to stabilize the cascade system $\Sigma-\Sigma_{f}$ on an equilibrium on which $x=0$.

It is worth noticing that the cascade $\Sigma-\Sigma_{f}$ can be seen as a system in feedforward form. The problem of stabilizing a feedforward system has attracted increasing interest by the control community and has been addressed with different tools: between the others, passivity approaches, see e.g. [22], state-feedback laws with satu- 
rated inputs, see e.g. [14,28] and state-feedback forwarding techniques, see e.g. [23, 24]. Based on forwarding, also an integral action approach can be applied, by folliwing [3]. The main weakness of the majority of these approaches, however, is that either the full-state $x$ needs to be known (or estimated) or a passivity assumption on the system $\Sigma_{f}$ is needed. In this paper, instead, we follow a different path and we explicitly use the information that the controller $\Sigma_{\eta}$ exists and has already been designed.

In particular, rather than designing an output feedback stabilizer for the cascade $\Sigma-\Sigma_{f}$, we propose to modify the known stabilizer $\Sigma_{\eta}$ by adding an opportune system which pre-processes the output $p$ of $\Sigma_{f}$. For this, the full knowledge of the state $x$ of the plant $\Sigma$ is not required. To this end, the main idea is to use a disturbance observer [8] designed in particular as an extended high-gain observer (see, e.g., [16,25], also known as extended state observer, [20]) able to recover the output $y$ of $\Sigma$ from the estimation of the state of the filter $\Sigma_{f}$. In the same spirit of standard results about separation principle for nonlinear systems in semi-global output feedback stabilization (see, e.g., [4, 29, 30]), the resulting controller allows to stabilize an equilibrium of the cascade system $\Sigma-\Sigma_{f}$ with a domain of attraction that includes the set of initial conditions. On this equilibrium, the state of $\Sigma$ coincides with the origin, thus recovering the properties of the original output feedback law $\Sigma_{\eta}$. The proposed approach can also be seen as a tool to design stabilization law for interconnected systems for which passivity conditions may not be satisfied and for which the design of a full-state observer may be impossible (or to be avoided). This, in particular, will be shown with an academic example.

Notation $\mathbb{R}$ is the set of real numbers, $\mathbb{R}_{\geq 0}:=[0, \infty)$, and $|\cdot|$ denotes the standard Euclidean norm in $\mathbb{R}^{n}$. Given $c>0$, we define $\mathbb{B}_{c}^{n}:=\left\{x \in \mathbb{R}^{n}:|x| \leq c\right\}$. If $n=1$, then we compactly write $\mathbb{B}_{c}$. Given $x \in \mathbb{R}^{n}, y \in$ $\mathbb{R}^{m}$, we denote $(x, y):=\left(x^{\top}, y^{\top}\right)^{\top}$. A triplet of matrices $\left(A_{n}, B_{n}, C_{n}\right)$ is in prime form of dimension $n$ if $A_{n} \in$ $\mathbb{R}^{n \times n}$ is a shift matrix (all 1's on the upper diagonal and all 0's elsewhere), $B_{n}^{\top}:=\left(\begin{array}{lll}0 \cdots 0 & 1\end{array}\right), B \in \mathbb{R}^{n \times 1}$ and $C_{n}:=\left(\begin{array}{lll}1 & 0 \cdots 0\end{array}\right) \in \mathbb{R}^{1 \times n}$. Given $s, L \in \mathbb{R}, L>0$, we define $\operatorname{sat}_{L}(s):=\min \{L, \max \{s,-L\}\}$. We refer to standard definitions of class $\mathcal{K}, \mathcal{K} \mathcal{L}$ functions [27].

\section{Main result}

We state the following assumptions.

Assumption 1 The functions $f, \phi, \psi$ in (1), (2), are locally Lipschitz and zero at the origin. The function $h$ is zero at the origin, and $d h / d x$ is locally Lipschitz. The triplet $\left(A_{r}, B_{r}, C_{r}\right)$ in (3) is in prime form and the function $q$ is locally Lipschitz and zero at the origin. The initial conditions of $(1),(2),(3)$, range in some given compact sets $X \times E \times Z \subset \mathbb{R}^{n} \times \mathbb{R}^{m} \times \mathbb{R}^{r}$.

Assumption 2 The origin of the closed-loop system (1), (2) is asymptotically stable with a domain of attraction $\mathcal{A} \supset X \times E$, and locally exponentially stable.

Assumption 3 One of the following is satisfied.

a. For any positive number $\mu>0$ and compact set $Z \subset$ $\mathbb{R}^{r}$, there exist a compact set $\bar{Z} \supseteq Z$ such that, for any continuous function $y:[0, \infty) \rightarrow \mathbb{B}_{\mu}$, solutions to $(3)$ satisfy $z(t) \in \bar{Z}$ for all $t \geq 0$.

b. System (3) is input-to-state stable (ISS) with respect to $y$, namely there exist a class $\mathcal{K}$ function $\gamma$ and a class $\mathcal{K} L$ function $\beta$ so that solutions to (3) satisfy $|z(t)| \leq \beta(|z(0)|, t)+\gamma\left(\sup _{s \in[0, t]}|y(s)|\right)$ for all $t \geq 0$.

Assumptions 1-2 state that all the functions in (1), (2) are smooth enough and that controller (2) asymptotically stabilizes the origin of the plant (1) with a prescribed domain of attraction $\mathcal{A}$. Assumption 3.a states that filter (3) satisfies a bounded-input bounded-state property (BIBS), while Assumption 3.b is a stronger condition, asking for an input-to-state (ISS) property [27]. In the main theorem below, we will analyze both the cases in which filter (3) satisfies either Assumption 3.a, or 3.b.

The goal of this paper is to design a control law of the form

$$
\dot{\zeta}=f_{c}(\zeta, p), \quad u=\psi_{c}(\zeta, p),
$$

achieving the stabilization of an equilibrium of the closed-loop system (1), (3), (4) on which $x=0$. Inspired by classical approaches in output feedback literature, we propose a simple paradigm composed of two steps. First, we design an extended high-gain observer $[8,16,25]$ able to recover the input $y$ from the filter $(3)$. Then, we replace the input of the controller (2) by the saturated version of the estimate given by the observer. By following this strategy, the state of controller (4) is then partitioned as $\zeta:=(\eta, \hat{z}, \sigma)$, where the dynamics of $\eta \in \mathbb{R}^{m}$ is given by

$$
\dot{\eta}=\phi\left(\eta, \operatorname{sat}_{L}(\sigma)\right), \quad u=\psi\left(\eta, \operatorname{sat}_{L}(\sigma)\right),
$$

with the functions $\phi, \psi$ defined as in (2), $L \in \mathbb{R}_{\geq 0}$ a positive parameter to be chosen large enough and initial conditions ranging in the compact set $E$ defined in Assumption 1 , and the dynamics of $(\hat{z}, \sigma) \in \mathbb{R}^{r} \times \mathbb{R}$ is given by

$$
\begin{aligned}
& \dot{\hat{z}}=A_{r} \hat{z}+B_{r}\left(\operatorname{sat}_{M}(q(\hat{z}))+\sigma\right)+D_{r}(g) K_{r}\left(p-C_{r} \hat{z}\right), \\
& \dot{\sigma}=g^{r+1} k_{r+1}\left(p-C_{r} \hat{z}\right),
\end{aligned}
$$


with initial conditions ranging in a compact set $R \subset \mathbb{R}^{r+1},\left(A_{r}, B_{r}, C_{r}\right)$ a triplet in prime form, $K_{r}:=\left(k_{1}, \ldots, k_{r}\right), D_{r}(g):=\operatorname{diag}\left(g, \ldots, g^{r}\right)$ with $g \geq 1$ being a (high-gain) positive parameter to be chosen large enough and $M \in \mathbb{R}_{\geq 0}$ a second positive parameter to be selected. We have the following result.

Theorem 1 Let $k_{1}, \ldots, k_{r+1}$ be chosen such that the characteristic polynomial $s^{r+1}+k_{1} s^{r}+\cdots+k_{r} s+k_{r+1}$ has all eigenvalues with strictly negative real part and let Assumption 1 and 2 hold. Then, there exist $L, M>0$, $g^{\star} \geq 1$ and $\delta \geq 0$, such that, for any $g>g^{\star}$ one of the following is satisfied.

- If Assumption 3.a holds, the solutions of the closedloop system (1), (3), (5), (6) converge asymptotically the set

$\mathcal{S}=\left\{(x, \eta, z, \hat{z}, \sigma): x=0, \eta=0,(z, \hat{z}) \in \mathbb{B}_{2 \delta}^{2 r}, \sigma=0\right\}$

for any initial condition in $X \times E \times Z \times R$.

- If Assumption $3 . b$ is satisfied, then $\delta$ in (7) is zero, namely the origin of the closed-loop system (1), (3), (5), (6) is asymptotically stable with a domain of attraction including $X \times E \times Z \times R$. If, in addition, the origin of (3) is locally exponentially stable, then the origin of the closed-loop system is also locally exponentially stable.

Proof Consider the change of coordinates $e_{i}:=$ $g^{r+1-i}\left(\hat{z}_{i}-z_{i}\right)$ for $i=1, \ldots, r$, and $e_{r+1}:=\sigma-h(x)$. The closed-loop system (1), (3), (5), reads

$$
\begin{aligned}
& \dot{\chi}=\varphi(\chi)+\Delta_{\varphi}(\chi, e) \\
& \dot{e}=g F e+\Delta_{\chi}(\chi, e)+\Delta_{z}(z, e) \\
& \dot{z}=A_{r} z+B_{r}(q(z)+h(\chi))
\end{aligned}
$$

where we used the compact notation $\chi:=(x, \eta), h(\chi):=$ $h(x)$, the function $\varphi, \Delta_{\varphi}, \Delta_{\chi}, \Delta_{z}$ are defined as

$$
\begin{aligned}
& \varphi(\chi):=(f(x, \psi(\eta, h(x))), \phi(\eta, h(x))) \\
& \varphi_{L}(\chi, e):=\left(f\left(x, \psi\left(\eta, \operatorname{sat}_{L}\left(h(x)+B_{r+1}^{\top} e\right)\right)\right),\right. \\
&\left.\phi\left(\eta, \operatorname{sat}_{L}\left(h(x)+B_{r+1}^{\top} e\right)\right)\right) \\
& \Delta_{\varphi}(\chi, e):=\varphi_{L}(\chi, e)-\varphi(\chi) \\
& \Delta_{\chi}(\chi, e):=-B_{r+1} \frac{\partial h(x)}{\partial x} \varphi_{L}(\chi, e), \\
& \Delta_{z}(z, e):=g H\left[\operatorname{sat}_{M}\left(q\left(z_{1}+\frac{e_{1}}{g^{r}}, \ldots, z_{r}+\frac{e_{r}}{g}\right)\right)\right. \\
&\left.-q\left(z_{1}, \ldots, z_{r}\right)\right]
\end{aligned}
$$

and $F:=\left(A_{r+1}-K_{r+1} C_{r+1}\right), H:=(B, 0), K_{r+1}:=$ $\left(k_{1}, \ldots, k_{r+1}\right)$, with $\left(A_{r+1}, B_{r+1}, C_{r+1}\right)$ being a triplet in prime form. Note that the initial conditions $(\chi(0), e(0), z(0))$ of $(8)$ ranges in the set $(X \times E) \times$ $\mathbb{B}_{d g^{r}}^{r+1} \times Z$, for some $d$ large enough. Since, by definition, $e=0$ implies $\sigma=0$ and $|\hat{z}-z|=0$, the proof of the theorem is completed if we are able to show that solutions of (8) converge to the set $\left\{\chi=0, e=0, z \in \mathbb{B}_{\delta}^{r}\right\}$, the latter implying also $(z, \hat{z}) \in \mathbb{B}_{2 \delta}^{2 r}$. To this end, we can follow most of the arguments used in the nonlinear separation principle in [4]. As a consequence, we sketch here the main steps and highlight the main differences with respect to [4].

Definition of the parameters. By using the converse Lyapunov theorem of Kurzwel, [19, Theorem 7], Assumption 2 allows to establish the existence of a Lyapunov function $V_{\chi}: \mathbb{R}^{n \times m} \rightarrow \mathbb{R}_{\geq 0}$ and some positive definite functions $\underline{\alpha}, \bar{\alpha}: \mathbb{R} \rightarrow \mathbb{R}_{\geq 0}$ and $\Phi: \mathbb{R}^{n \times m} \rightarrow \mathbb{R}_{\geq 0}$, satisfying

$$
\begin{gathered}
\underline{\alpha}(|\chi|) \leq V_{\chi}(\chi) \leq \bar{\alpha}(|\chi|), \quad \lim _{\chi \rightarrow \partial \mathcal{A}} \underline{\alpha}(|\chi|)=\infty \\
\frac{\partial V_{\chi}}{\partial \chi} \varphi(\chi) \leq-\Phi(\chi)
\end{gathered}
$$

for all $\chi \in \mathcal{A}$, where $\partial \mathcal{A}$ denotes the boundary of $\mathcal{A}$. In light of the properties (9), there exists $\overline{\mathrm{v}}_{\chi}>0$ such that the corresponding Lyapunov level set $\Gamma_{\chi}:=\{\chi$ : $\left.V_{\chi}(\chi) \leq \overline{\mathrm{v}}_{\chi}\right\}$ contains the set $(X \times E)$, namely $(X \times$ $E) \subset \Gamma_{\chi} \subset \mathcal{A}$. With this $\Gamma_{\chi}$ in mind, we can fix $L:=$ $\sup _{\chi \in \Gamma_{\chi}}|h(\chi)|$ and we denote we consider the set $\bar{Z}$ of Assumption 3.a corresponding to such $\mu=L$. We define then $M:=\sup _{z \in \bar{Z}}(q(z))$ and $\delta:=\sup _{z \in \bar{Z}}|z|$. With this choice we have fixed all the constants of the theorem, except from $g^{\star}$, that will be fixed later.

Existence of solutions. Recall that all functions are (at least) locally Lipschitz, see Assumption 1. As a consequence, solutions starting in $X \times E \times Z \times R$ are uniquely defined on some interval of time $[0, \bar{\tau})$. Since $(X \times E)$ is strictly contained in $\in \Gamma_{\chi}$, there exists $\tau \in(0, \bar{\tau})$ such that any solution to $(8)$ starting inside $(X \times E)$ satisfies $\chi(t) \in \Gamma_{\chi}$ for all $t \in[0, \tau]$. Then, we focus on the $e$-dynamics. By using standard arguments on high-gain observers, see for instance $[1,4,17]$, we can show that the value of $|e|$ can be made arbitrarily small in an arbitrary short amount of time by picking a value of $g$ large enough. This can be established by differentiating the Lyapunov function $V_{e}(e):=e^{\top} P e$ along solutions to (8), with $P=P^{\top}>0$ satisfying ${ }^{1} P F+F^{\top} P=-I$ (recall that $F$ is Hurwitz by construction). We obtain $\dot{V}_{e} \leq-g|e|^{2}+2 e^{\top} P\left(\Delta_{\chi}+\Delta_{z}\right)$. The following arguments can then be used: with $L$ fixed, there exists $\bar{\Delta}_{\chi} \in \mathbb{R}_{\geq 0}$ independent of $g$ so that $\left|\Delta_{\chi}(\chi, e)\right| \leq \bar{\Delta}_{\chi}$ for all $(\chi, e) \in$ $\Gamma_{\chi} \times \mathbb{R}^{r+1} ; \Delta_{z}(z, 0)=0$ for all $z \in \bar{Z}$; there exists $\ell_{z} \in \mathbb{R}_{\geq 0}$ independent of $g$ so that $\left|\Delta_{z}(z, e)\right| \leq \ell_{z}|e|$ for all $(\chi, \bar{e}) \in \Gamma_{\chi} \times \mathbb{R}^{r+1}$. Therefore, for any (arbitrarily small) $\varepsilon_{1}>0$, we can claim that there exists $g_{1}^{\star} \geq 1$ large enough, such that, for any $g>g_{1}^{\star}$ we have $|e(t)| \leq \varepsilon_{1}$ for all $t \in\left[\frac{\tau}{2}, \tau\right]$.

1 We denote with $\underline{p}, \bar{p}$ the smallest and the largest eigenvalues of $P$. 
Now consider the Lyapunov function $V_{\chi}$ defined in (9). Its derivative along solutions of (8) gives $\dot{V}_{\chi} \leq-\Phi(\chi)+$ $\left(\partial V_{\chi} / \partial \chi\right) \Delta_{\varphi}(\chi, e)$. Due to the saturation function in $\varphi_{L}$, there exists $\ell_{\varphi}$ satisfying $\left|\left(\partial V_{\chi} / \partial \chi\right) \Delta_{\varphi}(\chi, e)\right| \leq \ell_{\varphi}|e|$ for all $(x, e) \in \Gamma_{\chi} \times \mathbb{R}^{r+1}$. Since we can select $\varepsilon_{1}$ arbitrarily small, there exists $\varepsilon_{1}>0$ such that the derivative of $V_{\chi}$ along the $\chi$-dynamics in (8) satisfies $\dot{V}_{\chi} \leq 0$ for all $\chi \in \Gamma_{\chi}$ and all $|e| \leq \varepsilon_{1}$. This shows that $\chi(t) \in \Gamma_{\chi}$ for all $t \in[0, \tau]$. Note that this also implies $|h(\chi(t))| \leq L$ for all $t \in[0, \tau]$ and therefore, in light of Assumption 3.a, $z(t) \in \bar{Z}$ for all $t \in[0, \tau]$. In light of previous analysis, the set $\Gamma_{\chi} \times \mathbb{B}_{\varepsilon_{1}}^{r+1} \times \bar{Z}$ is invariant and therefore we can extend the solutions of $(8)$ to $[0, \infty)$. We deduce $(\chi(t), e(t), z(t)) \in \Gamma_{\chi} \times \mathbb{B}_{\varepsilon_{1}}^{r+1} \times \bar{Z}$ for all $t \in\left[\frac{\tau}{2}, \infty\right)$.

Practical Attractiveness. The second part of the proof consists in showing practical attractiveness of the equilibrium $(\chi, e)=0$. In particular, this can be established by applying the same arguments used in $[4$, Section $\mathrm{V}$ $\mathrm{B}]$ to the subsystem $(\chi, e)$. Note that in light of the Lipschitz properties of $\Delta_{z}$ and the fact that $\Delta_{z}(z, 0)=0$ for all $z \in \bar{Z}$, the system (8) can be viewed as a cascade between the subsystems $(\chi, e)$ and $z$. As a consequence, we can use the Laypunov functions $V_{\chi}(\chi)$ and $V_{e}(e)$ previously defined to show that for any arbitrarily small $\varepsilon_{2}>0$, there exists $T \geq \frac{\tau}{2}$ and $g_{2}^{\star} \geq g_{1}^{\star}$ large enough, such that $(\chi(t), e(t)) \in \mathbb{B}_{\varepsilon_{2}}^{n+m+r+1}$ for all $t \in[T, \infty)$, and for any $g>g_{2}^{\star}$.

Asymptotic Stability. Finally, the last part of the proof consists in showing the asymptotic convergence to the equilibrium $(\chi, e)=0$. For this, first we recall that the origin of $\dot{\chi}=\varphi(\chi)$ is locally exponentially stable, see Assumption 2, and invoke the converse Lyapunov theorem in [15, Theorem 4.14] to establish the existence of a Lyapunov function $\mathcal{V}_{\chi}(\chi)$, and real numbers $\underline{a}, \bar{a}, a_{0}, a_{1}, \varrho>0$ such that,

$$
\begin{gathered}
\underline{a}|\chi|^{2} \leq \mathcal{V}_{\chi}(\chi) \leq \bar{a}|\chi|^{2} \\
\frac{\partial \mathcal{V}_{\chi}}{\partial \chi} \varphi(\chi) \leq-a_{0}|\chi|^{2}, \quad\left|\frac{\partial \mathcal{V}_{\chi}}{\partial \chi}(\chi)\right| \leq a_{1}|\chi|
\end{gathered}
$$

for all $\chi \in \mathbb{B}_{\varrho}^{n+m}$. Select $\varepsilon_{2}=\varrho / 2$ (see the paragraph Practical Attractiveness), and define the Lyapunov function $\mathcal{W}(\chi, e):=\frac{1}{\bar{a}} \mathcal{V}_{\chi}(\chi)+\frac{1}{\bar{p}} V_{e}(e)$. By letting $\Omega_{\varrho}:=\{(\chi, e): \mathcal{W}(\chi, e) \leq \varrho\}$, we have $\mathbb{B}_{\varrho / 2}^{n+m+r+1} \subset \Omega_{\varrho}$ and $\sup _{\chi \in \Omega_{\varrho}}|\chi| \leq \varrho$. As a consequence, in light of previous arguments, for any $g>g_{2}^{\star}$, any solution $(\chi(t), e(t))$ to $(8)$ starting in $(X \times E) \times \mathbb{B}_{d g^{r}} \times Z$ satisfies $(\chi(T), e(T), z(T)) \in \Omega_{\varrho} \times \bar{Z}$. Now we compute the derivative of $\mathcal{W}$ as $\dot{\mathcal{W}} \leq-\frac{a_{0}}{\underline{a}}|\chi|^{2}-\frac{g}{\underline{p}}|e|^{2}+\beta(\chi, e, z)$ where $\beta(\chi, e, z):=\frac{1}{\bar{a}} \frac{\partial \mathcal{V}_{\chi}}{\partial \chi} \Delta_{\varphi}(\chi, e)+\frac{\overline{1}}{\bar{p}} \frac{\partial V_{e}}{\partial e}\left(\Delta_{\chi}(\chi, e)+\right.$ $\left.\Delta_{z}(z, e)\right)$. We recall that $\mathcal{V}_{\chi}, V_{e}, \Delta_{\varphi}, \Delta_{\chi}, \Delta_{z}$ are locally Lipschitz and moreover $\Delta_{\varphi}(\chi, 0)=0, \Delta_{\chi}(0,0)=0$,
$\Delta_{z}(z, 0)=0$. As a consequence by using the third inequality in (10), and by applying also Young's inequality, we can bound as $|\beta(\chi, e, z)| \leq \frac{c_{1}}{\kappa}|\chi|^{2}+\left(c_{2}+c_{2} \kappa\right)|e|^{2}$ for all $(\chi, e, z) \in \Omega_{\varrho} \times \bar{Z}$ for some constants $c_{1}, c_{2}, c_{3}>0$ independent of $g$ and any arbitrarily large $\kappa>0$. This gives $\dot{\mathcal{W}} \leq-\left(a_{0} / \underline{a}-c_{1} / \kappa\right)|\chi|^{2}-\left(g / \underline{p}-c_{2}(1+\kappa)\right)|e|^{2}$ for all $(\chi, e, z) \in \Omega_{\varrho} \times \bar{Z}$. By selecting $\kappa$ and $g^{\star} \geq g_{2}^{\star}$ larges enough, we obtain $\dot{\mathcal{W}} \leq-\left(c_{4}|\chi|^{2}+c_{5}|e|^{2}\right)$ for all $(\chi, e) \in \Omega_{\varrho}$, for some $c_{4}, c_{5}>0$, and for all $g>g_{3}^{\star}$. We conclude that all solutions satisfy $\lim _{t \rightarrow \infty}(\chi(t), e(t))=0$, namely all solutions converge asymptotically to $\left\{\chi=0, e=0, z \in \mathbb{B}_{\delta}^{r}\right\}$ (where we used the definition of $\delta$ given in Definition of the parameters). Furthermore, in view of the change of coordinates, $e=0$ implies $\sigma=0$ and $|\hat{z}-z|=0$, and therefore $(z, \hat{z}) \in \mathbb{B}_{2 \delta}^{r}$ which proves the first statement of Theorem 1. Similarly to [4], the second part of Theorem 1 can be directly obtained by using the ISS-property of Assumption 3.b and standard small-gain arguments for the interconnection of the subsystems $(\chi, e)$ and $z$.

\section{Remarks}

- Note that local exponential stability of the origin of (1)-(2) in Assumption 2 is, in our context, necessary to preserve asymptotic stability of the equilibrium $x=0$. When only local asymptotic stability is guaranteed, the proposed feedback design (5), (6) can guarantee only practical stability of $x=0$. In order to relax such assumption, a different design needs to be used.

- If the function $q$ in (3) is globally Lipschitz, then no saturation function in (6) is needed.

- The extended high-gain observer (6) may be designed by following the low-power high-gain observer paradigm introduced in $[1,2]$. Such design is of particular interest to address numerical challenges and improve the sensitivity to high-frequency measurement noise. We can therefore design an extended low-power high-gain observer, replacing (6), as

$$
\begin{gathered}
\dot{\xi}_{1}=A_{2} \xi_{1}+N_{2}^{\top} \xi_{2}+D_{2}(g) G_{1}\left(p-C_{2} \xi_{1}\right), \\
\dot{\xi}_{i}=A_{2} \xi_{i}+N_{2}^{\top} \xi_{i+1}+D_{2}(g) G_{i}\left(B_{2}^{\top} \xi_{i-1}-C_{2} \xi_{i}\right), \\
i=2, \ldots, r-2, \\
\dot{\xi}_{r-1}=A_{2} \xi_{r-1}+N_{2}^{\top} \xi_{r}+B_{2} \operatorname{sat}_{M}(q(\hat{z})) \\
\quad+D_{2}(g) G_{r-1}\left(B_{2}^{\top} \xi_{r-2}-C_{2} \xi_{r-1}\right), \\
\dot{\xi}_{r}=A_{2} \xi_{r}+C_{2}^{\top} \operatorname{sat}_{M}(q(\hat{z})) \\
+D_{2}(g) G_{r}\left(B_{2}^{\top} \xi_{r-1}-C_{2} \xi_{r}\right), \\
\sigma=B_{2}^{\top} \xi_{r},
\end{gathered}
$$

where $\xi=\left(\xi_{1}, \ldots, \xi_{r}\right) \in \mathbb{R}^{2 r}$ is the full state of the observer, with $\xi_{i} \in \mathbb{R}^{2}$, for $i=1, \ldots, r, N_{2}=B_{2} B_{2}^{\top}$, $\left(A_{2}, B_{2}, C_{2}\right)$ is a triplet in prime form of dimension $2, D_{2}(g)=\operatorname{diag}\left(g, g^{2}\right)$, and $G_{i} \in \mathbb{R}^{2 \times 1}, i=1, \ldots, r$, 
are some fixed coefficients to be selected. In view of the properties of the observer (11) highlighted in [1, 2 ], the result of Theorem 1 holds for the closed-loop system (1), (3), (5), (11) if the coefficients $G_{1}, \ldots, G_{r}$ are chosen accordingly to [1, Lemma 1]. Moreover, the peaking-phenomenon, see [17], can be removed by following the saturation design proposed in [2].

\section{Example}

Results of Theorem 1 can be applied also to design stabilizing output feedback laws for interconnected systems that do not satisfy passivity conditions [22]. Consider for instance the following nonlinear system

$$
\begin{aligned}
\dot{w}_{1} & =\rho\left(w_{1}\right)+w_{2}+u, & & \dot{w}_{3}=w_{4}, \\
\dot{w}_{2} & =-w_{1}+w_{2}^{2} u, & & \dot{w}_{4}=w_{1}+w_{2}^{3}-w_{3}-w_{4}, \\
p & =w_{3}, & &
\end{aligned}
$$

where $w:=\left(w_{1}, w_{2}, w_{3}, w_{4}\right) \in \mathbb{R}^{4}$ is the state, with initial condition ranging in some given compact set $W \subset$ $\mathbb{R}^{4}, u \in \mathbb{R}$ is the control input, $p \in \mathbb{R}$ is the measured output, and $\rho(\cdot)$ is any Lipschitz function satisfying $\rho(s) s \leq 0$ for any $s \in \mathbb{R}$. Our objective is to design an output feedback law of the form (4) to stabilize the origin of (12). First, define $x:=\left(w_{1}, w_{2}\right), z:=\left(w_{3}, w_{4}\right)$ so that we obtain the representation $(1),(3)$ in which

$$
\begin{gathered}
f(x, u)=\left(\begin{array}{c}
\phi\left(x_{1}\right)+x_{2}+u \\
-x_{1}+x_{2}^{2} u
\end{array}\right), \quad h(x)=x_{1}+x_{2}^{3}, \\
q(z)=-z_{1}-z_{2}=Q z, \quad Q=(-1,-1) .
\end{gathered}
$$

The $x$-subsystem (1), (13) is passive with input $u$ and output $y=w_{1}+w_{2}^{3}$, and zero-state detectable. This can be established by means of the Lyapunov function $V\left(w_{1}, w_{2}\right):=\frac{1}{2}\left(w_{1}^{2}+w_{2}^{2}\right)$, and by noting that $\dot{V}=$ $w_{1} \rho\left(w_{1}\right)+\left(w_{1}+w_{2}^{3}\right) u \leq y u$. As a consequence (see for instance [15]), Assumption 2 can be verified for system (1), (13), by selecting for instance the state feedback law $u=\psi(y)$ with $\psi$ any function satisfying $s \psi(s)<0$ for any $s \neq 0$. For example, $\psi(s)=-\kappa s$ for some $\kappa>0$. On the other hand, $z$-subsystem(3), (14) with input $y$ and output $p$ is linear but not passive, since the relative degree between $y$ and $p$ is 2 (see [15, Example 6.4], [15, Lemma 6.4]). Nevertheless, since the the poles of $A_{2}+B_{2} Q$ have strictly negative real part, Assumptions 3.b can be easily verified by using the Lyapunov function $V_{z}(z)=z^{\top} P_{z} z$, with $P_{z}=P_{z}^{\top}>0$ satisfying $P_{z}\left(A_{2}+B_{2} Q\right)+\left(A_{2}+B_{2} Q\right)^{\top} P_{z} \leq-\lambda I$ for some $\lambda>0$. As a consequence, we can apply Theorem 1 to design a dynamic semi-global output feedback law for system (12) by designing the dynamic controller $\hat{z}, \sigma$ given by (6) and by selecting $u=\psi\left(\operatorname{sat}_{L}(\sigma)\right)$ for some $L>0$ large enough.

\section{Conclusion}

In this note, we considered the problem of designing stabilizing feedback laws for nonlinear systems with filtered output. Given an output feedback control law which satisfies some stability requirements, we showed that, in place of a complete redesign, adding an extended high-gain observer allows to retrieve the desired stability properties for the system. With an eye to the application in power networks, this result can be further extended to the multi-agent framework to solve the problem of synchronization when dynamics over the communication line is present, see [6].

\section{References}

[1] D. Astolfi and L. Marconi. A high-gain nonlinear observer with limited gain power. IEEE Transactions on Automatic Control, 60(11):3059-3064, 2015.

[2] D. Astolfi, L. Marconi, L. Praly, and A. R. Teel. Low-power peaking-free high-gain observers. Automatica, 98:169-179, 2018.

[3] D. Astolfi and L. Praly. Integral action in output feedback for multi-input multi-output nonlinear systems. IEEE Transactions on Automatic Control, 62(4):1559-1574, 2017.

[4] A. N. Atassi and H. K. Khalil. A separation principle for the stabilization of a class of nonlinear systems. IEEE Transactions on Automatic Control, 44(9):1672-1687, 1999.

[5] U. Borup, F. Blaabjerg, and P. N. Enjeti. Sharing of nonlinear load in parallel-connected three-phase converters. IEEE Transactions on Industry Applications, 37(6):18171823, 2001.

[6] G. Casadei, L. Marconi, and C. De Persis. Synchronization of nonlinear oscillators over networks with dynamic links. In IEEE 54th Annual Conference on Decision and Control, pages 6184-6189, 2015.

[7] C. Chen, C. Qian, Z. Sun, and Y. Liang. Global output feedback stabilization of a class of nonlinear systems with unknown measurement sensitivity. IEEE Transactions on Automatic Control, 63(7):2212-2217, 2018.

[8] W. Chen, J. Yang, L. Guo, and S. Li. Disturbanceobserver-based control and related methods - an overview. IEEE Transactions on Industrial Electronics, 63(2):1083$1095,2016$.

[9] N. Ghods and M. Krstic. Source seeking with very slow or drifting sensors. Journal of Dynamic Systems, Measurement, and Control, 133(4):044504, 2011.

[10] D. Groß, M. Colombino, JS. Brouillon, and F. Dörfler. The effect of transmission-line dynamics on grid-forming dispatchable virtual oscillator control. IEEE Transactions on Control of Network Systems, 2019.

[11] W. P. M. H. Heemels and N. van De Wouw. Stability and stabilization of networked control systems. In Networked Control Systems, pages 203-253. Springer, 2010.

[12] A. Hierlemann and R. Gutierrez-Osuna. Higher-order chemical sensing. Chemical reviews, 108(2):563-613, 2008.

[13] A. Isidori. Nonlinear control systems. Springer Science \& Business Media, 2013.

[14] G. Kaliora and A. Astolfi. Nonlinear control of feedforward systems with bounded signals. IEEE Transactions on Automatic Control, 49(11):1975-1990, 2004. 
[15] H. K. Khalil. Noninear systems. Pearson, 2002.

[16] H. K. Khalil. Extended high-gain observers as disturbance estimators. SICE Journal of Control, Measurement, and System Integration, 10(3):125-134, 2017.

[17] H. K. Khalil and L. Praly. High-gain observers in nonlinear feedback control. International Journal of Robust and Nonlinear Control, 24(6):993-1015, 2014.

[18] M. Krstic, I. Kanellakopoulos, P. V. Kokotovic, et al. Nonlinear and adaptive control design, volume 222. Wiley New York, 1995.

[19] J. Kurzwel. On the inversion of lyapunov's second theorem on stability of motion. AMS Translations Series 2, 24:19-77, 1963.

[20] S. Li, J. Yang, W. Chen, and X. Chen. Generalized extended state observer based control for systems with mismatched uncertainties. IEEE Transactions on Industrial Electronics, 59(12):4792-4802, 2012.

[21] Z. Lin and T. Hu. Semi-global stabilization of linear systems subject to output saturation. Systems \& Control Letters, 43(3):211-217, 2001.

[22] R. Lozano, N. Brogliato, and I.D. Landau. Passivity and global stabilization of cascaded nonlinear systems. IEEE Transactions on Automatic Control, 37(9):1386-1388, 1992.

[23] F. Mazenc. Interconnected nonlinear systems, local and global stabilization. Systems \& control letters, 35(5):317-323, 1998.

[24] F. Mazenc and L. Praly. Adding integrations, saturated controls, and stabilization for feedforward systems. IEEE Transactions on Automatic Control, 41(11):1559-1578, 1996.

[25] S. Nazrulla and H. K. Khalil. Robust stabilization of nonminimum phase nonlinear systems using extended highgain observers. IEEE Transactions on Automatic Control, 56(4):802-813, 2011.

[26] D. Nesic and D. Liberzon. A unified framework for design and analysis of networked and quantized control systems. IEEE Transactions on Automatic control, 54(4):732-747, 2009.

[27] E. D. Sontag. Input to state stability: Basic concepts and results. In Nonlinear and optimal control theory, pages 163220. Springer, 2008.

[28] A. R. Teel. A nonlinear small gain theorem for the analysis of control systems with saturation. IEEE transactions on Automatic Control, 41(9):1256-1270, 1996.

[29] A. R. Teel and L. Praly. Global stabilizability and observability imply semi-global stabilizability by output feedback. Systems \& Control Letters, 22(5):313-325, 1994.

[30] A. R. Teel and L. Praly. Tools for semiglobal stabilization by partial state and output feedback. SIAM Journal on Control and Optimization, 33(5):1443-1488, 1995. 